\title{
Perinephric hematoma induced by factor Xa inhibitor in a patient with a vascular renal mass
}

\author{
Syed A. Hussaini, Abdul Rahim Ali Bakhsh \\ Department of Internal Medicine, Zayed Military Hospital, Abu Dhabi, United Arab Emirates
}

\begin{abstract}
Perinephric hematomas are known to present in the form of Lenk's triad with acute flank pain, flank mass and hypovolemic shock. Here, we describe a case of perinephric hematoma occurring secondary to the use of anticoagulant therapy in the setting of a renal mass. To the best of our knowledge, this is the first reported case of a perinephric hematoma occurring secondary to the use of Apixaban. The patient was an 80 year old male with a history of the presence of a left sided vascular renal mass discovered seven years ago admitted from a peripheral health center with pneumonia and a dropping hemoglobin along with acute kidney injury. Evaluation of his course revealed the use of a Factor Xa inhibitor, namely Apixaban, for new onset atrial fibrillation. The patient was stabilized with multiple units of packed red blood cell transfusions. An abdominal computed tomography abdomen demonstrated a perinephric hematoma contained in the Gerotas fascia. Due to deranged renal function, the patient was managed conservatively and made a full recovery. This case highlights the challenges associated with the diagnosis of perinephric bleeds. The use of anticoagulation therapy in the setting of a pre-existing vascular lesion remains a dilemma.
\end{abstract}

Key words: perinephric hematoma, apixaban, NOACs, renal mass

\section{INTRODUCTION}

Perinephric hematomas are well known to occur in a wide variety of settings. These bleeds are classically described to present in the form of Lenk's triad with acute flank pain, flank mass and hypovolemic shock. ${ }^{[1]}$ Whilst some of these hematomas are reported to occur spontaneously, ${ }^{[1]}$ secondary causes such as renal tumors, ${ }^{[2]}$ the use of anticoagulant therapy ${ }^{[3]}$ and procedural complications of renal biopsy ${ }^{[4]}$ are also frequently reported. Amongst renal tumors, angiomyolipomas and renal cell carcinomas are known to be most frequently associated with perinephric hematomas. ${ }^{[1]}$ The course of such hemorrhages varies from full recovery with conservative management to the need for nephrectomy, emergent laparotomy, IR guided embolization to even death. Here, we describe a case of a patient with a vascular renal mass, who on initiation of apixaban for paroxysmal atrial fibrillation developed a large peri-renal hematoma.

\section{CASE REPORT}

The patient was an 80 -year-old male with a past medical history of CVA with residual left hemiparesis and stable chronic kidney disease stage 3 and a history of a left renal mass discovered on a routine ultrasound abdomen nearly 7 years ago. The patient had undergone CT Abdomen with IV contrast at that time for further evaluation where the mass was noted to be heterogeneously enhancing $2.7 \mathrm{~cm} \times 2.4 \mathrm{~cm}$ in dimension arising from the cortex of the mid pole of the left kidney and hyper-vascular in nature. The patient was advised to undergo a partial nephrectomy, which he had declined. The mass had remained stable on subsequent follow up imaging.

The patient was admitted to our hospital 
from a nearby health facility with a diagnosis of severe pneumonia requiring ICU hospitalization. Upon admission, he was diagnosed with anemia with a hemoglobin of $7.5 \mathrm{~g} / \mathrm{dL}$, which dropped further requiring up to 5 units of PRBC transfusion. He was also having acute kidney injury on top of chronic kidney disease with a creatinine of $442 \mathrm{mg} / \mathrm{dL}\left(\mathrm{eGFR} 10 \mathrm{~mL} / \mathrm{min} / 1.73 \mathrm{~m}^{2}\right)$. On review of his course in the previous healthcare facility, it was discovered that the patient had developed atrial fibrillation, which was cardioverted pharmacologically with amiodarone. He was subsequently maintained in sinus rhythm with bisoprolol. As he had a high CHA2DS2-VASc score, anticoagulation was initiated with a Non Vitamin K oral anticoagulant (NOAC) - Apixaban. His hemoglobin soon dropped to $4.9 \mathrm{~g} / \mathrm{dL}$ requiring up to 4 units of packed red blood cell transfusions for resuscitation. There was no bleeding per rectum or melena and all other sources of blood loss were ruled out.

The patient required another 3 units of packed red cell transfusions in our hospital after admission. He subsequently underwent an ultrasound abdomen that revealed a significant collection around the left kidney (Figure 1). A CT abdomen without contrast was arranged due to his $A K I$ and it revealed a $10 \mathrm{~cm} \times 8 \mathrm{~cm}$ perinephric hematoma, which had completely distorted the renal mass and the cortex (Figure 2).
The anticoagulation was soon held and a decision to conservatively manage the hematoma was made by a multidisciplinary team including urologists and interventional radiologists. The patient recovered from his pneumonia and recovered his renal function. He maintained a stable hemoglobin and a subsequent follow up imaging demonstrated the hematoma to be unchanged in size but contained within the perinephric fascia.

\section{DISCUSSION}

Perinephric hematomas are a discrete entity of life threatening medical emergencies where the diagnosis is often made later in the course often on the background of clinical suspicion, signs of developing shock and a fall in hemoglobin. ${ }^{[1]}$ It is not unheard of the first diagnosis of these hemorrhages to be made on autopsy. ${ }^{[5]}$ Renal tumors are considered to be the most common cause of these bleeds, with angiomyolipoma often being considered as the most frequent cause. ${ }^{[1]}$ This was also the case with our patient who had a heterogeneous mass in his left kidney for the past 6 years. The clear nature of this mass was not known to us due to the lack of a consistent follow up by the patient and insufficient diagnostic studies.

The use of anticoagulant therapy is also another cause that has been reported to trigger these bleeds. ${ }^{[3]}$ Interestingly,

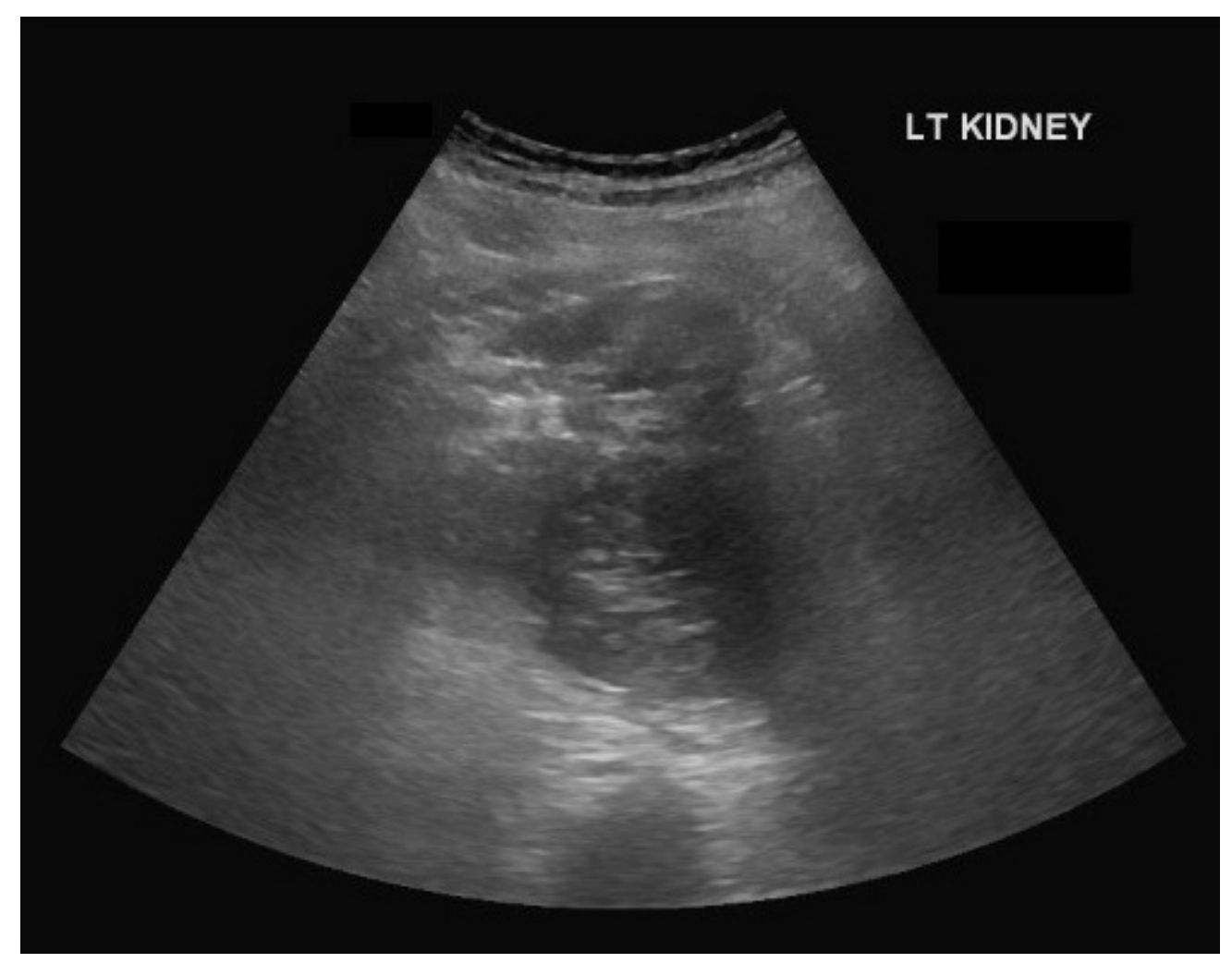

Figure 1: US KUB showing left kidney with loss of corticomedullary differentiation with a mass of mixed echogenicity. 


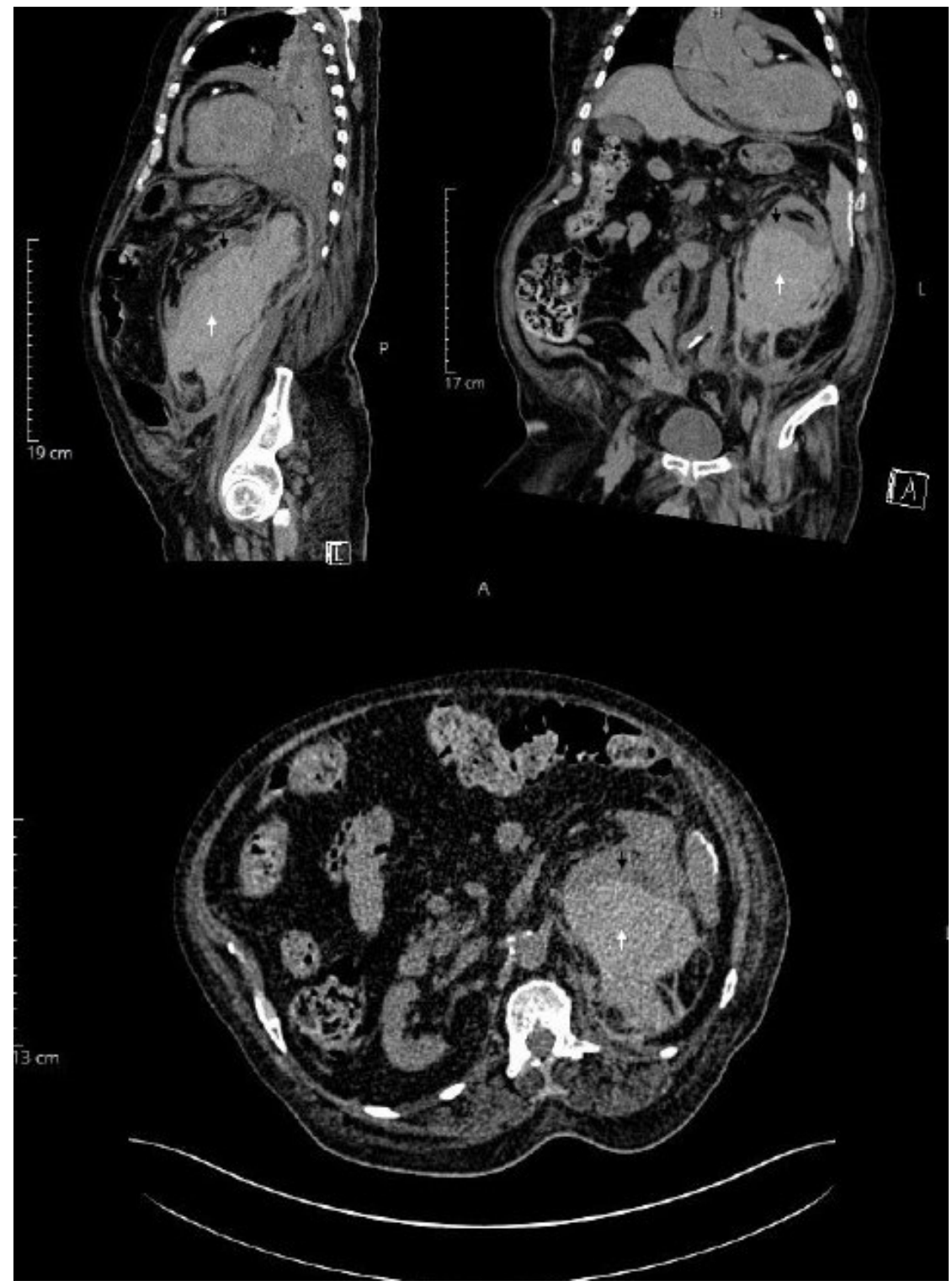

Figure 2: CT abdomen (non-contrast) showing hematoma in the renal mass (white arrow) with distortion of the renal cortex (black arrow) in the sagittal (top-left), coronal (top-right) and transverse (bottom) planes.

on review of literature, we found only one case report of a spontaneous perinephric hematoma developing secondary to the use of a NOAC, namely dabigatran. ${ }^{[6]}$ Our case had a similar history of prior use of a NOAC, apixaban for paroxysmal atrial fibrillation. At present, there are no reported cases of a spontaneous perinephric hematoma developing in the setting of the use of apixaban. Another interesting aspect our case highlights is the simultaneous presence of a renal mass and the use of anticoagulation. In the setting of the need for anticoagulation, such as a DVT or atrial fibrillation, this case highlights a therapeutic dilemma. In addition to the existing benefit and risk estimation, such as the use of CHA2DS2-VASc and HAS-BLED scores for atrial fibrillation, perhaps further knowledge of the nature of these pre-existing renal lesions such as their size, vascularity, Bosniak classification and so on, may also need to be considered when deliberating the use of anticoagulation. In the simultaneous presence of such a lesion, it could be wiser to consider the use of warfarin or a heparin based anticoagulant, which can be reversed easily, rapidly and cost-effectively should the need arise.

The treatment of perinephric hematomas depends largely on the clinical status of the patient. In an acute presentation, stabilization of the patient and emergent transfusions with close monitoring of the hemoglobin and hematocrit are the mainstay. There are a number of approaches to manage this condition, although no clear evidence based guidelines exist regarding this issue at present. In the absence of any known 
tumor and a normal contralateral kidney, nephrectomy of the affected kidney followed by a careful pathological evaluation of the specimen has been advised by some authors, as there is noted to be an increased incidence of the presence of small tumors in such cases. ${ }^{[7]}$ The presence of a lesion other than the hematoma in the kidney is itself an indication for nephrectomy according to some authors. ${ }^{[8]}$ Radiologically guided intervention is a relatively safe procedure with good reported outcomes and has taken preference over surgery. ${ }^{[0]}$

A number of reports have also described a conservative approach of observation and supplemental transfusions to successfully manage this condition. ${ }^{[10]}$ This is particularly convenient due to the often simultaneous presence of an element of acute kidney injury, often caused due to the hemorrhage itself, which precludes the use of contrast based imaging in certain cases. This was also a dilemma in our case where despite the presence of a vascular mass, the bleed was managed conservatively due to poor underlying renal function, advanced age and multiple co-morbidities. A very close observation of the patient is the key when taking this approach.

\section{CONCLUSION}

We described a case of a perinephric hematoma, which had developed on the setting of a vascular renal mass on the initiation of anticoagulation, which was managed conservatively and made a full recovery. The use of anticoagulation therapy in the setting of a pre-existing vascular lesion remains a dilemma.

\section{Conflict of Interest}

The authors of this manuscript declare no conflicts of interest.

\section{REFERENCES}

1. Wang B, Pureza V, Wang H. A tale of Wunderlich syndrome. J Surg Case Rep 2012;2012:rjs015.

2. Wright $\mathrm{T}$, Sooriakumaran P. Renal angiomyolipoma presenting with massive retroperitoneal haemorrhage due to deranged clotting factors: a case report. Cases J 2008;1:213.

3. Greco M, Butticè S, Benedetto F, Spinelli F, Traxer O, Tefik T, et al. Spontaneous Subcapsular Renal Hematoma: Strange Case in an Anticoagulated Patient with HWMH after Aortic and Iliac Endovascular Stenting Procedure. Case Rep Urol 2016;2016: 2573476.

4. Adjei-Gyamfi Y, Koffman G, Amies T, Easty M, Marks S, McHugh K. Reversible acute anuric kidney injury after surgical evacuation of perinephric hematomas as a complication of renal transplant biopsies. Pediatr Transplant 2014;18:E262-5.

5. Durak D, Eren F, Inanir N, Eren B, Çetin S, Gündoğmuş ÜN. Spontaneous Rupture of a Renal Cell Carcinoma Associated with Fatal Bleeding. Maedica (Buchar) 2014;9:275-7.

6. Parajuli S, Saxena N. Spontaneous Perinephric Hematoma with Newer Oral Anticoagulation in Kidney Transplant Recipient. J Nephrol Renal Ther 2016; 2: 002.

7. Kendall AR, Senay BA, Coll ME. Spontaneous subcapsular renal hematoma: Diagnosis and management. J Urol 1988;139:246-50.

8. Morgentaler A, Belville JS, Tumeh SS, Richie JP, Loughlin KR. Rational approach to evaluation and management of spontaneous perirenal hemorrhage. Surg Gynecol Obstet 1990;170:121-5.

9. Jain V, Ganpule A, Vyas J, Muthu V, Sabnis RB, Rajapurkar MM, et al. Management of non-neoplastic renal hemorrhage by transarterial embolization. Urology 2009;74:522-6.

10. Sotošek S, Markić D, Španjol J, Krpina K, Knežević S, Maričić A. Bilateral Wünderlich Syndrome Caused by Spontaneous Rupture of Renal Angiomyolipomas. Case Rep Urol 2015;2015: 316956.

How to cite this article: Hussaini SA, Bakhsh ARA. Perinephric hematoma induced by factor $\mathrm{Xa}$ inhibitor in a patient with a vascular renal mass. J TransI Intern Med 2020; 8: 195-8. 